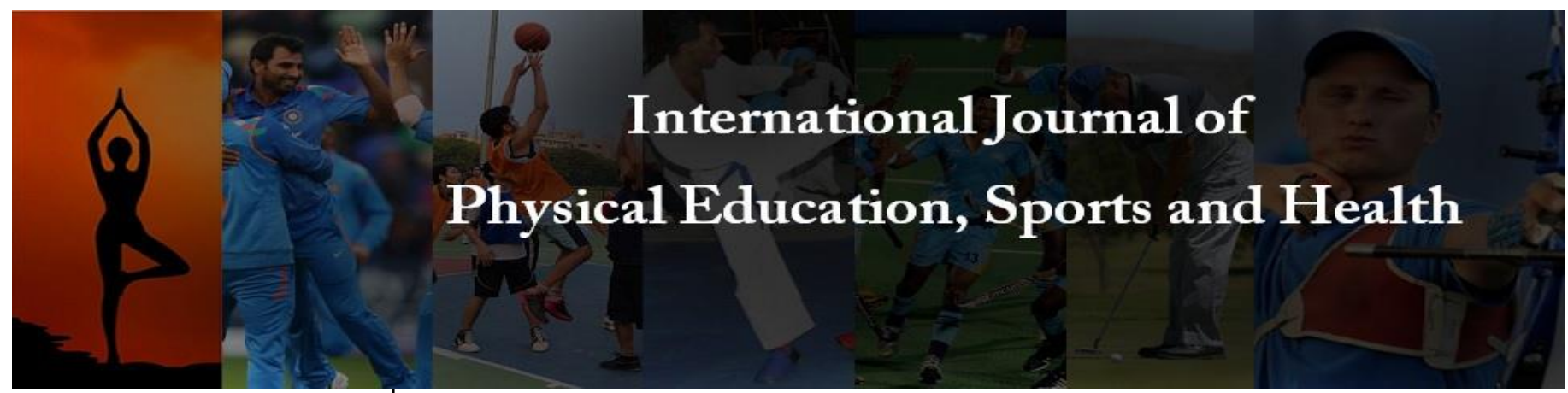

P-ISSN: 2394-1685

E-ISSN: 2394-1693

Impact Factor (ISRA): 5.38

IJPESH 2020; 7(4): 275-278

(C) 2020 IJPESH

www.kheljournal.com

Received: 08-05-2020

Accepted: 17-06-2020

Cyanna Joseph D'souza

MPT, Lecturer, Laxmi Memorial

College of Physiotherapy,

Mangalore, Karnataka, India

PV Abhilash

MPT, Associate Professor,

Laxmi Memorial College of

Physiotherapy, Mangalore,

Karnataka, India

Nripesh Nepal

BPT, Laxmi Memorial College of

Physiotherapy, Mangalore,

Karnataka, India

\section{The effect of positional release technique on upper trapezius myofascial trigger points: A case series}

\author{
Cyanna Joseph D'souza, PV Abhilash and Nripesh Nepal
}

DOI: https://doi.org/10.22271/kheljournal.2020.v7.i4e.1808

\section{Abstract}

Objective: This study aimed to determine the effectiveness of the Positional Release Therapy (PRT) for treating Myofascial Trigger Points (MTrPs) in the upper trapezius muscle on outcomes of pain, Range of Motion (ROM) and disability.

Methods: Twenty-one patients of mean age 29 \pm 12.48 years were screened for inclusion/exclusion criteria. The PRT was administered three times a week for 4 weeks. The verbal Numerical Pain Rating scale (NPRS), active cervical contralateral flexion (ACLF) and Neck Disability Index (NDI) were recorded at baseline, 2 and 4 weeks. Descriptive statistics in the form of mean and standard deviation were used to analyze the data.

Results and Conclusion: Increase in ROM and alleviated levels of pain and disability were noted in all participants post treatment. This case series suggests that a short-term manual therapy technique, such as the PRT would be beneficial in treating individuals with upper trapezius TrPs.

Keywords: Case series, positional release therapy, upper trapezius, trigger points, manual therapy

\section{Introduction}

Myofascial pain syndrome, a relevant musculoskeletal disorder, is most commonly known to occur due to repetitive tasks with long hours of static load, and is currently reported to affect nearly $85 \%$ of the population at some point in life ${ }^{[1,2]}$. It is characterized by the presence of Trigger Points (TrPs) which are small, hyperalgesic spots within the connective tissue that refer pain to distant sites ${ }^{[3]}$. TrPs can be active or latent. Latent TrPs cause no pain until irritated by intense heat or cold. On the other hand, active TrPs can produce constant pain, decreased muscle tone, strength and range of motion (ROM), thus leading to disability ${ }^{[4]}$.

TrPs can occur in any muscle, but it is usually seen to occur in muscles that help maintain posture ${ }^{[5]}$. Trester et al. reported the most commonly involved muscle is the upper trapezius, and can be a potential contributing factor in conditions like non-specific neck pain and tension headaches where it is difficult to identify the pathoanatomical source of an individual's pain ${ }^{[6}$, 7,8]. The exact mechanism of formation of a TrP is unknown. According to Harden et al., TrPs that are associated with an end-plate disorder and increased release of acetylcholine, results in local ischemia and sensitization of nociceptors ${ }^{[9]}$. An increased release of inflammatory chemical substances including prostaglandins, histamine, serotonin and bradykinin is observed at the TrP site affecting the membrane of polymodal nociceptive receptors. This in turn causes peripheral sensitization, causing central sensitization and chronic pain ${ }^{[9,10]}$. Saavedra et al. showed that the EMG signal of the muscle with TrPs generates a greater change in the electrical signal in the resting position when compared to a normal muscle ${ }^{[11]}$.

Various therapeutic approaches are available for the treatment of TrPs, among which manual therapy is considered highly effective ${ }^{[12]}$. It is known as a non-invasive treatment which can be used in conjunction with several electrical modalities ${ }^{[3]}$. One such manual therapy technique, used for treatment of TrPs, is the Positional Release Therapy (PRT). It involves passive body positioning, which is claimed to elicit immediate and prolonged reductions in pain and tenderness. PRT relies on precise positioning of dysfunctional tissues in ways that allow a spontaneous response reducing excessive tension or spasm ${ }^{[13]}$.
Corresponding Author: MPT, Lecturer, Laxmi Memoria College of Physiotherapy, Mangalore, Karnataka, India 
Several studies have reported a reduction in $\mathrm{TrP}$ symptoms after its management by different procedures, especially muscle energy techniques and myofascial release therapy, but the effectiveness of PRT in remains unclear ${ }^{[14,15]}$. Therefore, the objective of the present study was to analyze the influence of PRT in patients with upper trapezius MTrPs on outcomes of pain, ROM and disability.

\section{Materials and Methods \\ 2.1 Participants}

In this case series, a total of 21 patients visiting a tertiary hospital in Dakshina Karnataka with a present condition of unilateral upper trapezius MTrPs were recruited. An informed consent was obtained from all participants once they had been screened for suitability and any relevant questions were answered on the procedure and data collection process.

\subsection{Screening process protocol and rationale}

At their first visit, all participants were screened for inclusion/exclusion criteria. Inclusion criteria- patients between ages 18 to 35 years irrespective of gender with active upper trapezius MTrPs or non-specific neck pain (radiating or non-radiating) without any specific systematic disease being detected as the underlying cause of the complaints and less than 3 months duration. Patients responding with a cry, grimace or wince to palpation of the upper trapezius muscle called the 'jump sign'. Exclusion criteria- neck symptoms related to a motor vehicle collision or significant trauma, signs of serious pathology (e.g. malignancy, infection, inflammation, or fracture), signs of cervical spinal cord compromise (e.g. diffuse sensory abnormality, diffuse weakness, hyperreflexia, or the presence of clonus), two or more signs of nerve root involvement (e.g. dermatomal sensation changes, myotomal weakness, or diminished/absent tendon jerk reflexes), history of neck surgery in the past 12 months, history of cervical degenerative joint diseases, endocrine disorders, and autoimmune conditions (e.g. rheumatoid arthritis, fibromyalgia, etc.) or received trigger point injections in the upper trapezius muscle within the past 6 months.

\subsection{Baseline assessment and key outcome measures}

Subjective measures (verbal Numerical Pain Rating Scale (NPRS) and Neck Disability Index (NDI)) and objective outcome measure (active cervical contralateral flexion (ACLF)) were recorded for each participant at the first baseline assessment and thereafter at intervals of 2 weeks and 4 weeks.

There is evidence to support the construct validity of the verbal NPRS ${ }^{[16]}$. For this, participants indicated the intensity of pain by reporting a number that best represented it, between 0 (no pain) and 10 (maximum pain) ${ }^{[17]}$. The NDI is a 10-item questionnaire which has shown to be a valid and reliable measure of disability in individuals with neck pain (ICC 50.70-0.89) ${ }^{[18]}$. It is scored from $0-50$ points $(0-100 \%)$ in which higher scores correspond to greater levels of disability ${ }^{[18]}$. ACLF was assessed using a universal goniometer which has demonstrated good to excellent interrater reliability ${ }^{[19]}$. The shoulder was stabilized to arrest elevation. Subjects were asked to sit upright and laterally flex their head towards the opposite side of involvement and the motion was stopped once the available ROM was completed. Each subject performed three test trials and the average was used for analysis.

\subsection{PRT protocol}

With the subject in seated position, the therapist stood posterior to the subject's shoulder ${ }^{[20]}$. The therapist the palpated for the tender point using a pincer grasp that produced a jump sign. The subject's arm was taken for flexion, abduction and external rotation with the head flexed to the treated side. This position of ease was maintained for about 90 seconds ${ }^{[20]}$. The technique was repeated thrice and the subject was slowly passively placed in neutral position of the cervical spine in between each trial.

All participants were treated three times per week for 4 consecutive weeks and were instructed to maintain their normal activities while avoiding any undue stress to the neck.

\subsection{Data analysis}

Statistical package SPSS (IBM SPSS Statistics for Windows, ver. 21.0. Armonk, NY: IBM Corp.) was used to analyze the data. Descriptive statistics were used to explore the data and included the mean and Standard Deviation (SD) at baseline, 2 weeks and 4 weeks.

\section{Results and Discussion}

Twenty-one participants with active upper trapezius MTrPs or non-specific neck pain were recruited (12 females and 9 males); mean \pm SD age and BMI $29 \pm 12.48$ years and $24.96 \pm 7.32 \mathrm{~kg} / \mathrm{m}^{2}$ respectively. At baseline there were a total of 28 active MTrPs between 21 participants, on discharge no active MTrPs were found in the upper trapezius.

There was a significant improvement in the outcome measures from baseline to 2 and 4 weeks (Table 1 and 2). A mean difference of approximately 5 points in verbal NPRS between baseline assessment and on discharge was found. On discharge the difference in ACLF indicated a mean increase of about $14^{\circ}$. Similarly, all participants reported an overall increase in NDI scores. The mean change in NDI at 4 weeks post discharge was around $35 \%$ (Table 2).

These results suggest that application of PRT can improve outcomes of pain, ROM and disability. These findings are consistent with the results of Shawabka et al. who demonstrated that trigger point sensitivity reduces in response to a single application of PRT ${ }^{[21]}$. Saavedra et al. also found the PRT to be an effective alternative for relieving pain and reducing baseline EMG signals in the upper trapezius muscle with a $\operatorname{TrP}{ }^{[11]}$. Our findings also came in agreement with Maseguer et al. who found that the PRT was beneficial in reducing tenderness represented by an increase in pressure pain thresholds of trigger points in the upper trapezius muscle of subjects with mechanical neck pain ${ }^{[22]}$. The PRT has also proved to be effective in individuals with tender points of low back pain, sports injuries of the upper extremity and tension headaches ${ }^{[23-25]}$.

The application of PRT is claimed to reduce unusual firing from the site of muscular irritation. ${ }^{[20]}$ PRT theoretically corrects neuromuscular hyperirritability and muscular hyper tonicity and reduces tissue tension allowing for the resolution of the inflammatory response and the release of the fascial restriction ${ }^{[26]}$. Based on previous literature, it appears that PRT techniques have the capacity to provide immediate relief of tenderness and local pain provoked by TrPs ${ }^{[21,27]}$.

The limitations of this study include a lack of comparison group. Reliability issues on physical examination and manual palpation of TrP diagnosis are reported by systematic reviews thus, creating a need for high TrP palpation/diagnostic studies $[28,29]$. All data collection and interventions were performed by 
the same individual, posing a risk for researcher bias. Although the authors found significant reductions in the outcomes, these findings require a higher level of change to be considered clinically meaningful. Due to the short term follow up, the findings cannot be generalized, thus recommending a longer follow up period to future researchers in further determine the lasting effect of the approach.

Table 1: Mean scores and SD of outcome measures

\begin{tabular}{|c|c|c|c|}
\hline Outcome Measure & Baseline & 2 weeks & 4 weeks \\
\hline NPRS & $7.40 \pm 0.52$ & $4.60 \pm 0.70$ & $1.60 \pm 2.01$ \\
\hline ACLF (in degrees) & $24.70 \pm 4.30$ & $31.90 \pm 4.04$ & $39.00 \pm 12.46$ \\
\hline NDI (percentage) & $46.70 \pm 11.52$ & $22.80 \pm 8.28$ & $11.40 \pm 18.55$ \\
\hline
\end{tabular}

SD: Standard Deviation; NPRS: Numerical Pain Rating Scale;

ACLF: Active Cervical Contralateral Flexion; NDI: Neck Disability Index

Table 2: Change scores from baseline to 2 and 4 weeks

\begin{tabular}{|c|c|c|}
\hline Outcome Measure & $\begin{array}{c}\text { Difference from } \\
\text { baseline assessment } \\
\text { to 2 weeks }\end{array}$ & $\begin{array}{c}\text { Difference from } \\
\text { baseline assessment } \\
\text { to 4 weeks }\end{array}$ \\
\hline NPRS & $2.80 \pm 1.30$ & $5.80 \pm 2.04$ \\
\hline ACLF (in degrees) & $7.20 \pm 1.87$ & $14.30 \pm 1.20$ \\
\hline NDI (percentage) & $23.80 \pm 8.51$ & $35.30 \pm 7.40$ \\
\hline
\end{tabular}

SD: Standard Deviation; NPRS: Numerical Pain Rating Scale;

ACLF: Active Cervical Contralateral Flexion; NDI: Neck Disability Index

\section{Conclusion}

Manual interventions such as the PRT can be useful to alleviating pain, improving range of motion and functional ability in patients with TrPs in the upper trapezius muscle. These findings contribute to the growing evidence and use of manual therapy in musculoskeletal pain syndromes. Results of this study should be accepted by caution given the reliability issues in physical examination and diagnosis of TrPs. Apart from the identified limitations, this case series will inform more rigorous investigations in this area of research.

\section{References}

1. Huguenin LK. Myofascial trigger points: the current evidence. Physical Ther Sport. 2004; 5(1):2-12.

2. Tulder MV, Malmivaara A, Koes B. Repetitive strain injury. Lancet. 2007; 369(9575):1815-1822.

3. Simons DG, Travel J, Simons LS. Myofascial Pain and Dysfunction: The Trigger Point Manual. 2nd ed, Vol. 1. Upper Half of Body. Williams and Wilkins, Baltimore, 1999.

4. Harden RN, Bruehl SP, Gass S, Niemiec C, Barbick B. Signs and symptoms of the myofascial pain syndrome: A national survey of pain management providers. Clin $\mathbf{J}$ Pain. 2000; 16(1):64-72.

5. Lavelle ED, Lavelle W, Smith HS. Myofascial trigger points. Anesthesiol Clin. 2007; 25(4):841-3.

6. Trester D, Marras WS, Burr D, Sheedy JE, Hart D. Myofascial trigger point development from visual and postural stressors during computer work. J Electromyogr Kinesiol. 2006; 16(2):115-24.

7. Borghouts JA, Koes BW, Bouter LM. The clinical course and prognostic factors of non-specific neck pain: a systematic review. Pain. 1998; 77(1):1-13.

8. Davidoff R. Trigger points and myofascial pain: toward understanding how they affect headaches. Cephalalgia. 1998; 18(7):436.

9. Harden RN, Cottrill J, Gagnon CM, Smitherman TA, Weinland SR, Tann B et al. Botulinum toxin A in the treatment of chronic tension-type headache with cervical myofascial trigger points: a randomized, double-blind, placebo controlled pilot study. Headache. 2009; 49(5):732-43.

10. Hwang M, Kang YK, Kim DH. Referred pain pattern of the pronator quadratus muscle. Pain. 2005; 116(3):23842.

11. Saavedra FJ, Cordeiro MT, Alves JV, Fernandes HM, Reis VM, Mont'Alverne DG. The influence of positional release therapy on the myofascial tension of the upper trapezius muscle. Rev Bras Cineantropom Desempenho Hum. 2014; 16(2):191-99.

12. Alvarez D, Rockwell P. Trigger points: diagnosis and management. Am Fam Physician. 2002; 65(4):653-60.

13. Chaitow L. Muscle Energy \& Positional Release Technique. Techniques, 2005.

14. Chaitow L. Modern neuromuscular techniques. Churchill Livingstone, Edinburgh, 1996.

15. Chaitow L. Neuro-muscular technique. Thorsons, Wellingborough, 1982.

16. Briggs M, Closs JS. A descriptive study of the use of visual analogue scales and verbal rating scales for the assessment of postoperative pain in orthopaedic patients. J Pain Symptom Manage. 1999; 18(6):438-46.

17. Marquie L, Duarte LR, Marine C, Lauque D, Sorum PC. How patients and physicians rate patients' pain in a French emergency department using a verbally administered numerical rating scale and a visual analog scale. Acute Pain. 2008; 10(1):31-7.

18. Vernon H, Mior S. The neck disability index - A study of reliability and validity. J Manipulative Physiol Ther. $1991 ; 14(7): 409-15$.

19. Youdas JW, Carey JR, Garrett TR. Reliability of measurements of cervical spine range of motion comparison of three methods. Phys Ther. 1991; 71(2):98106.

20. Umashankar Mohanty. Clinical symposia in manual therapy. Ed 1. MTFI Healthcare Publications. India, 2017.

21. AL-Shawabka SA, Shenouda MM, Balbaa AA. Positional Release Technique Versus Manual Pressure Release on the Upper Trapezius Muscle in Patients with Myofascial Pain Dysfunction Syndrome. Bull Fac Ph Th Cairo Univ. 2013; 18(1):55-63.

22. Meseguer AA, Fernandez-de-las Penas C, Navarro-Poza JL, Rodriguez Blanco C, Gandia JJB. Immediate effects of the strain/counterstrain technique in local pain evoked by tender points in the upper trapezius muscle. Clin Chiropr. 2006; 9(3):112-8.

23. Hutchinson R. An investigation into the efficacy of strain- counterstrain technique to produce immediate changes in pressure pain thresholds in symptomatic subjects. School of Health Science Dissertations and Theses, 2008.

24. Bendtsen L, Jensen R, Jensen NK, Olesen J. Muscle palpation with controlled finger pressure: new equipment for the study of tender myofascial tissues. 1994; 59(2):235-9.

25. Ghanbari A, Rahimijaberi A, Mohamadi M, Abbasi, Sarvestani FK. The effect of trigger point management by positional release therapy on tension type headache. Neurorehabil. 2012; 30(4):333-9.

26. D'Ambrogio K, Roth G. Positional release therapy. Mosby, St. Louis, 1997.

27. Weiselfish S. Manual Therapy for the Orthopedic and 
Neurologic Patient Emphasizing Strain and Counterstrain Technique. Hartford, CT, Regional Physical Therapy (self-published), 1993.

28. Lucas N, Macaskill P, Irwig I, Moran R, Bogduk N. Reliability of physical examination for diagnosis of myofascial trigger points: a systematic review of literature. Clin J Pain. 2009; 25(1):80-9.

29. Myburgh C, Larsen AH, Hartvigsen J. A systematic critical review of manual palpation for identifying myofascial trigger points: evidence and clinical significance. Archives of Phys Med Rehabil. 2008; 89(6):1169-76. 